\title{
A divergence free fractional-step method for the Navier-Stokes equations on non-staggered grids
}
S. W. Armfield ${ }^{1}$
N. Williamson ${ }^{2}$
M. P. Kirkpatrick ${ }^{3}$
R. Street ${ }^{4}$

(Received 31 January 2010; revised 1 September 2010)

\begin{abstract}
The Navier-Stokes equations may be discretised using finite-volume schemes on non-staggered or on staggered grids. Non-staggered grid schemes typically require a derivation that leads to an error in the divergence to prevent the occurrence of oscillations in the pressure field with accompanying problems in integration. The staggered grid does not have the problem of pressure oscillations; however, this is at the expense of increased code complexity. Two standard non-staggered schemes are compared to a modified scheme in which the divergence error does not occur.
\end{abstract}

http://anziamj . austms .org.au/ojs/index.php/ANZIAMJ/article/view/2627 gives this article, (c) Austral. Mathematical Soc. 2010. Published September 22, 2010. ISSN 1446-8735. (Print two pages per sheet of paper.) Copies of this article must not be made otherwise available on the internet; instead link directly to this URL for this article. 


\section{Contents}

1 Introduction

C655

2 Method

C657

2.1 Divergence error . . . . . . . . . . . . . . . . . C658

2.2 Modified sparse scheme . . . . . . . . . . . . . . C661

3 Results and discussion

C662

4 Conclusions

C663

References

C666

\section{Introduction}

Non-staggered grids store all the unknowns at the same locations, as shown in Figure 1 where both non-staggered and staggered grid configurations are shown. In the past, many non-staggered grid Navier-Stokes solvers have been found to suffer from grid-scale oscillations in the pressure field that adversely affect their performance [1]. A number of approaches prevent the pressure oscillations, all of which effectively modify the continuity equation by the inclusion of a biharmonic pressure term $[1,2,3,4,5]$. Such an approach removes the problem of pressure oscillations at the expense of the divergence error associated with the inclusion of the biharmonic operator. These schemes were all derived in the context of iterative solvers.

Staggered grid schemes automatically couple the grid scale pressure to the remainder of the solution, and thus do not suffer from the problem of pressure oscillation [1]. Figure 1 shows the layout of the standard staggered grid, where the velocities are stored at locations offset from the pressure storage locations in their respective directions. The staggered scheme does have a number of disadvantages when compared to the non-staggered scheme. Each 

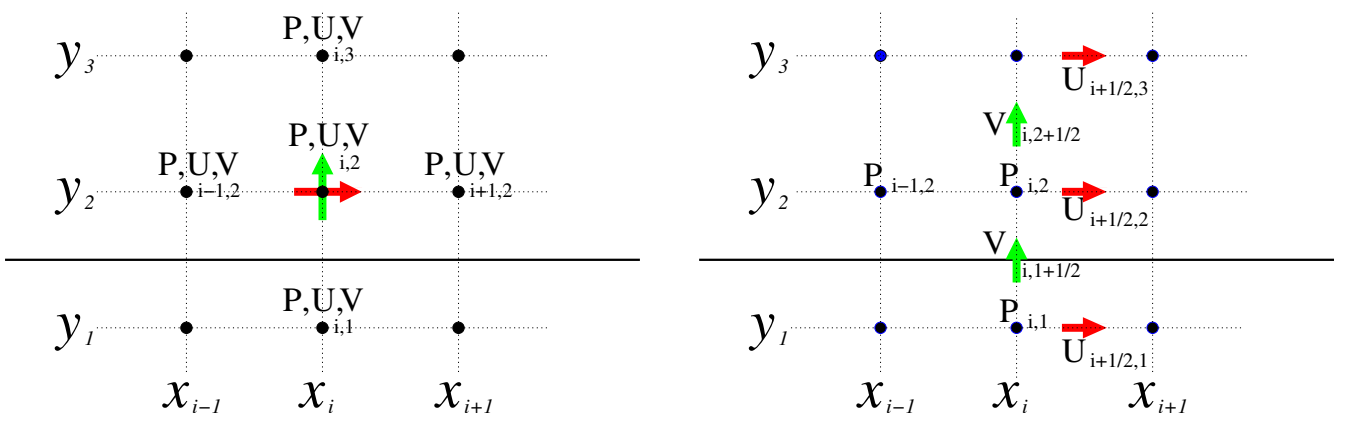

FiguRE 1: Non-staggered grid (left), staggered grid (right).

of the velocity components and the pressure have separate control volumes, leading to more complex coding, and additional interpolations are needed to obtain quantities on control volume boundaries.

Fractional step, non-iterative, non-staggered grid Navier-Stokes solvers typically use a compact discretisation for the pressure correction Poisson's equation, leading to an error in the divergence equation, similar to that of the iterative solvers cited above, that is independent of the accuracy of the solution of the Poisson's equation. The use of a sparse discretisation for the Poisson's equation will theoretically ensure a divergence free velocity field, but the accumulation of grid scale error in the pressure field can degrade the performance of the scheme. A sparse Poisson's equation is used to solve for the full pressure field, rather than the pressure correction field, thereby preventing the accumulation of grid scale error in the pressure field. This approach is shown to work well, and allows the effect of the divergence error in the compact approach to be studied. The performance of the schemes is examined in the context of turbulent duct flow. 


\section{Method}

Two non-staggered grid schemes are presented below. The standard staggered grid scheme is similiar and the differences are noted. The governing equations are the three-dimensional Navier-Stokes equations

$$
\begin{aligned}
\mathfrak{u}_{\mathrm{t}}+(\mathfrak{u} \cdot \nabla) \mathfrak{u} & =-\nabla \mathrm{P}+\frac{1}{\operatorname{Re}} \nabla^{2} \mathrm{u}, \\
\nabla \cdot \mathfrak{u} & =0,
\end{aligned}
$$

where $u$ is the velocity, $P$ the kinematic pressure and Re the Reynolds number. The Reynolds number is defined as $\mathrm{Re}=\mathbf{U H} / 2 \boldsymbol{v}$ with $\mathbf{U}$ the mean friction velocity and $H$ the duct height.

Equations (1) and (2) are discretised in time using second-order AdamsBashforth for the advective terms and Crank-Nicolson for the diffusive terms. For the momentum and continuity equations this gives the system

$$
\begin{aligned}
\frac{v^{\mathrm{n}+1}-v^{\mathrm{n}}}{\Delta \mathrm{t}}+\left[\frac{3}{2} \mathrm{~A}\left(v^{\mathrm{n}}\right)-\frac{1}{2} \mathrm{~A}\left(v^{\mathrm{n}-1}\right)\right] & =-\mathrm{G} \mathrm{p}^{\mathrm{n}+1 / 2}+\frac{1}{2 \operatorname{Re}} \mathrm{L}\left(v^{\mathrm{n}+1}+v^{\mathrm{n}}\right), \\
\mathrm{D} v^{\mathrm{n}+1} & =0
\end{aligned}
$$

where $(v, p)$ are the discrete velocity and pressure respectively, $A$ is the discrete advection operator, $\mathrm{G}$ the discrete gradient, $\mathrm{L}$ the discrete Laplace operator, and $\mathrm{D}$ the discrete divergence. Standard second order, central difference, discretisations are used for the operators A, G, L and D. Equation (3) is a second order in time representation of equation (1) at the $n+1 / 2$ time location.

Fractional-step methods integrate equations (3) and (4) in a segregated manner; that is, the momentum equations are first solved for the velocity, and some form of Poisson's equation is then solved for the pressure. The Poisson's equation is constructed from the momentum equation and the continuity equation and, as well as providing the pressure, acts to enforce continuity. 
In the standard method equation (3) is solved, using the best current value for $p$, to obtain $v^{*}$, an approximation to $v^{\mathrm{n}+1}$, that is

$$
\frac{v^{*}-v^{n}}{\Delta t}+\left[\frac{3}{2} A\left(v^{n}\right)-\frac{1}{2} A\left(v^{n-1}\right)\right]=-G p^{n-1 / 2}+\frac{1}{2 \operatorname{Re}} \mathrm{L}\left(v^{*}+v^{n}\right),
$$

where the $n-1 / 2$ time-level pressure is that obtained at the previous time step. This approximate velocity will not initially satisfy continuity. A correction is then applied of the form

$$
v^{\mathrm{n}+1}=v^{*}-\Delta \mathrm{tG} \pi
$$

where $\pi$ is a pressure correction, such that the resulting $v^{\mathfrak{n}+1}$ does satisfy continuity. An equation for $\pi$ is constructed by substituting equation (6) into the continuity equation (4), to give

$$
\mathrm{L} \pi=\mathrm{D} v^{*} / \Delta \mathrm{t}
$$

where $L=D G$, and the discrete form of $L$ used in this equation is discussed below.

Once the correction $\pi$ is obtained, the pressure is updated as

$$
p^{n+1 / 2}=p^{n-1 / 2}+\pi
$$

The $v^{*}$ field is then corrected as above and the integration continues to the next time step.

\subsection{Divergence error}

On a non-staggered grid using the standard approach the discrete Poisson's equation for $\pi$ is

$$
\left(\frac{\pi^{i+1, j}-2 \pi^{i, j}+\pi^{i-1, j}}{\Delta x^{2}}\right)+\left(\frac{\pi^{i, j+1}-2 \pi^{i, j}+\pi^{i, j-1}}{\Delta y^{2}}\right)
$$




$$
=\frac{1}{\Delta t}\left(\frac{\mathrm{U}^{i+1 / 2, j}-\mathrm{U}^{i-1 / 2, j}}{\Delta x}+\frac{\mathrm{V}^{i, j+1 / 2}-\mathrm{V}^{\mathrm{i}, \mathrm{j}-1 / 2}}{\Delta \mathrm{y}}\right)^{*},
$$

where to clarify the discussion the equation is written in two dimensional $(x, y)$ coordinate form with $\mathrm{U}$ and $\mathrm{V}$ the $\mathrm{x}$ and $\mathrm{y}$ components of velocity. This Poisson's equation is constructed by discretising the continuous form of equation (7) using centred, second order, discretisations. On the non-staggered grid the.$^{i \pm 1 / 2}$ and ${ }^{j \pm 1 / 2}$ values are obtained by linear interpolation from the nodal values. On the staggered grid these values are available and no interpolation is required.

The use of the compact discretisation, given in equation (9), introduces an error into continuity for the non-staggered scheme, which may be seen by substituting the corrected form of the velocity into the continuity equation. In this case

$$
\begin{aligned}
& u^{i, j, n+1}=u^{i, j *}-\Delta t\left(\frac{\pi^{i+1, j}-\pi^{i-1, j}}{2 \Delta x}\right), \\
& u^{i+1 / 2, j, n+1}=\left(\frac{U^{i+1, j, n+1}+U^{i, j, n+1}}{2}\right),
\end{aligned}
$$

and similarly for the other terms, giving

$$
\begin{aligned}
& \left(\frac{U^{i+1 / 2, j}-U^{i-1 / 2, j}}{\Delta x}+\frac{V^{i, j+1 / 2}-V^{i, j-1 / 2}}{\Delta y}\right)^{n+1} \\
& =\Delta t\left[\left(\frac{\pi^{i+1, j}-2 \pi^{i, j}+\pi^{i-1, j}}{\Delta x^{2}}+\frac{\pi^{i, j+1}-2 \pi^{i, j}+\pi^{i, j-1}}{\Delta y^{2}}\right)\right. \\
& \left.-\left(\frac{\pi^{i+2, j}-2 \pi^{i, j}+\pi^{i-2, j}}{4 \Delta x^{2}}+\frac{\pi^{i, j+2}-2 \pi^{i, j}+\pi^{i, j-2}}{4 \Delta y^{2}}\right)\right] .
\end{aligned}
$$

The right-hand side error term is the difference between sparse and compact discretisations of the Laplace operator. The sparse discretisation has a computational molecule with $(2 \Delta x, 2 \Delta y)$ spacing between the nodes, while the compact discretisation has $(\Delta x, \Delta y)$ spacing, as seen here. This structure 
results from the use of a compact Laplace operator in equation (9), and to second order the error

$$
\text { Error } \sim \Delta t\left[\Delta x^{2} \pi_{x x x x}+\Delta y^{2} \pi_{y y y y}\right] .
$$

Thus, using the compact Laplacian leads to a divergence error proportional to the fourth spatial derivative of the pressure correction $\pi$. Noting that $\pi \sim \Delta t p_{\mathrm{t}}$ means that this error is second order in time,

$$
\text { Error } \sim \Delta t^{2}\left[\Delta x^{2} p_{t x x x x}+\Delta y^{2} p_{t y y y y}\right] .
$$

The advantage of the staggered grid scheme, in comparison to the nonstaggered grid, is that when the Laplace operator for $\pi$ is constructed directly from the discrete forms of the divergence and gradient operators, a compact discretisation is obtained which does not produce a divergence error.

The use of the compact Laplace operator with the non-staggered scheme has been found to perform well $[6,7]$. The compact operator minimises the grid-scale error in $\pi$; however, the pressure field itself is still uncoupled at the grid scale and may accumulate grid-scale error. A commonly used modification to the compact non-staggered scheme that fully couples the grid scale pressure into the solution is the pressure weighted interpolation scheme of Rhie and Chow [5], whereby the ${ }^{i \pm 1 / 2}$ and.$^{j \pm 1 / 2}$ velocity fields for use in the divergence source term in the pressure correction Poisson's equation are obtained as

$$
\begin{aligned}
u^{i+1 / 2 *}= & \frac{1}{2}\left[\left(u^{i+1 *}+\Delta t \frac{\left(p^{i+2}-p^{i}\right)}{2 \Delta x}\right)+\left(u^{i *}+\Delta t \frac{\left(p^{i+1}-p^{i-1}\right)}{2 \Delta x}\right)\right] \\
& -\Delta t \frac{\left(p^{i+1}-p^{i}\right)}{\Delta x}
\end{aligned}
$$

and similarly for $\mathrm{V}^{*}$. This approach leads to a divergence error in the corrected velocity field of the same form as that in the standard compact scheme, but which is now first order in time:

$$
\text { Error } \sim \Delta t\left[\Delta x^{2} p_{x x x x}+\Delta y^{2} p_{y y y y}\right] .
$$




\subsection{Modified sparse scheme}

Constructing the discrete form of the Laplace operator directly from the discrete forms of the divergence and gradient operators on the non-staggered grid, with centred, second order, differences used for D and G gives a sparse discrete Laplace operator of the form

$$
\left(\frac{\pi^{i+2, j}-2 \pi^{i, j}+\pi^{i-2, j}}{4 \Delta x^{2}}\right)+\left(\frac{\pi^{i, j+2}-2 \pi^{i, j}+\pi^{i, j-2}}{4 \Delta y^{2}}\right) .
$$

The use of this sparse operator approach produces no divergence error but has been found to be inefficient and to lead directly to the pressure oscillations observed in iterative non-staggered schemes.

The modified sparse scheme adds the pressure gradient to the velocity field prior to the solution of the Poisson's equation, approximately cancelling the pressure gradient included in the momentum equations:

$$
\widehat{v}^{*}=v^{*}+\Delta \mathrm{tG} p^{n-1 / 2} .
$$

A sparse Poisson's equation is then solved for the full pressure at the next time step; that is,

$$
\begin{aligned}
& \left(\frac{p^{i+2, j}-2 p^{i, j}+p^{i-2, j}}{4 \Delta x^{2}}\right)^{n+1 / 2}+\left(\frac{p^{i, j+2}-2 p^{i, j}+p^{i, j-2}}{4 \Delta y^{2}}\right)^{n+1 / 2} \\
& =\frac{1}{\Delta t}\left(\frac{U^{i+1 / 2, j}-U^{i-1 / 2, j}}{\Delta x}+\frac{V^{i, j+1 / 2}-V^{i, j-1 / 2}}{\Delta y}\right)^{*} .
\end{aligned}
$$

With the $v^{n+1}$ velocity then obtained from the modified $v^{*}$ as above, but using the total pressure; that is,

$$
v^{\mathrm{n}+1}=\widehat{v}^{*}-\Delta \mathrm{tG} \mathrm{p}^{\mathrm{n}+1 / 2} .
$$

The corrected velocity will then be divergence free in proportion to the accuracy with which the pressure Poisson's equation is solved. The advantage 
TABLE 1: Variation of divergence error with residual.

\begin{tabular}{llllll}
\hline $\mathrm{p} / \pi$ residual & 100 & 10 & 1.0 & 0.1 & 0.01 \\
\hline Divergence error & & & & & \\
Compact & 0.7 & 0.69 & 0.68 & 0.65 & 0.65 \\
Compact+Rhie-Chow & 7.0 & 6.8 & 6.7 & 6.5 & 6.5 \\
Sparse modified & 0.1 & 0.01 & 0.001 & 0.0001 & 0.00001 \\
\hline
\end{tabular}

of the modified sparse scheme is that the pressure is calculated anew at each time step, and grid scale oscillations in the pressure field will not accumulate. This approach was found to be most efficient if the pressure passed to the Poisson solver was initialised using the pressure from the previous time step.

\section{Results and discussion}

Results have been obtained for turbulent duct flow in the domain $0 \leqslant x \leqslant$ $6.3,0 \leqslant y \leqslant 2.0,0 \leqslant z \leqslant 3.15$ with periodic boundary conditions in $x$ and $z$, and zero-slip wall boundary conditions in $y$. The flow is driven by a constant streamwise pressure gradient in the $x$ direction, $\partial \mathrm{P} / \partial x=1.0$ with the Reynolds number $R e=180$ (with Re based on friction velocity $\mathbf{U}$, equal to $\left.\operatorname{Re}_{\tau}[8]\right)$. This configuration corresponds to that given by Moser et al. [8] where a spectral method provided the solution used here as the benchmark for the schemes considered. A grid with uniform $\Delta x=0.204, \Delta z=0.1016$ and non-uniform $\Delta y$ is used $(\Delta y=0.014$ at the walls with a stretching rate of 1.07 moving away from the walls), giving a total grid-size of $51 \times 56 \times 36$ in the $x, y, z$ directions respectively. The time step is $\Delta t=0.001$ given a maximum Courant number of 0.25. The maximum cell Reynolds number is $800\left(\operatorname{Re}_{\text {cell }}=\max (\mathrm{U} \Delta \mathrm{x} \operatorname{Re}, \mathrm{V} \Delta \mathrm{y} R \mathrm{R})\right)$.

Table 1 shows the dependence of the divergence error on the accuracy of the solution of the $\pi$ and $p$ Poisson's equations. In this case the divergence error is the integral of the absolute local value of the divergence over the 
domain. As can be seen solving the Poisson's equation to greater accuracy does not reduce the divergence error for the two compact schemes, as a result of the error terms described above. Additionally the divergence error is approximately an order of magnitude larger for the compact scheme with the Rhie-Chow interpolation. In contrast, the divergence error for the sparse scheme is reduced in proportion to the accuracy with which the $p$ Poisson's equation is solved.

Figure 2 contains results for the time-averaged streamwise velocity profiles compared to the benchmark spectral result with $\mathrm{U}$ the $x$ component of the instantaneous velocity. There is some variation in the accuracy of the schemes, with Rhie-Chow the least accurate and the standard compact and modified sparse schemes having approximately the same accuracy. Results for the $\overline{\mathrm{U}^{\prime} \mathrm{U}^{\prime}}$ component of the Reynolds stress are shown in Figure 3, with again Rhie-Chow the least accurate of the schemes and, again, little difference between the standard compact and the modified sparse schemes.

\section{Conclusions}

Both compact schemes and the modifed sparse scheme provide a reasonable approximation to the benchmark spectral results on a relatively coarse mesh. The Rhie-Chow scheme is the least accurate, presumably as a result of the increased divergence error. The divergence error of the standard compact scheme is not having a significant adverse effect on the solution, when compared to the sparse modified scheme. At 800 the cell Reynolds number is considerably greater than the normal requirement of less than two for linear stability for a centred difference scheme. It may be that in a strongly unsteady flow of this type the non-linear effects are sufficient to control the solution and prevent unbounded growth. 


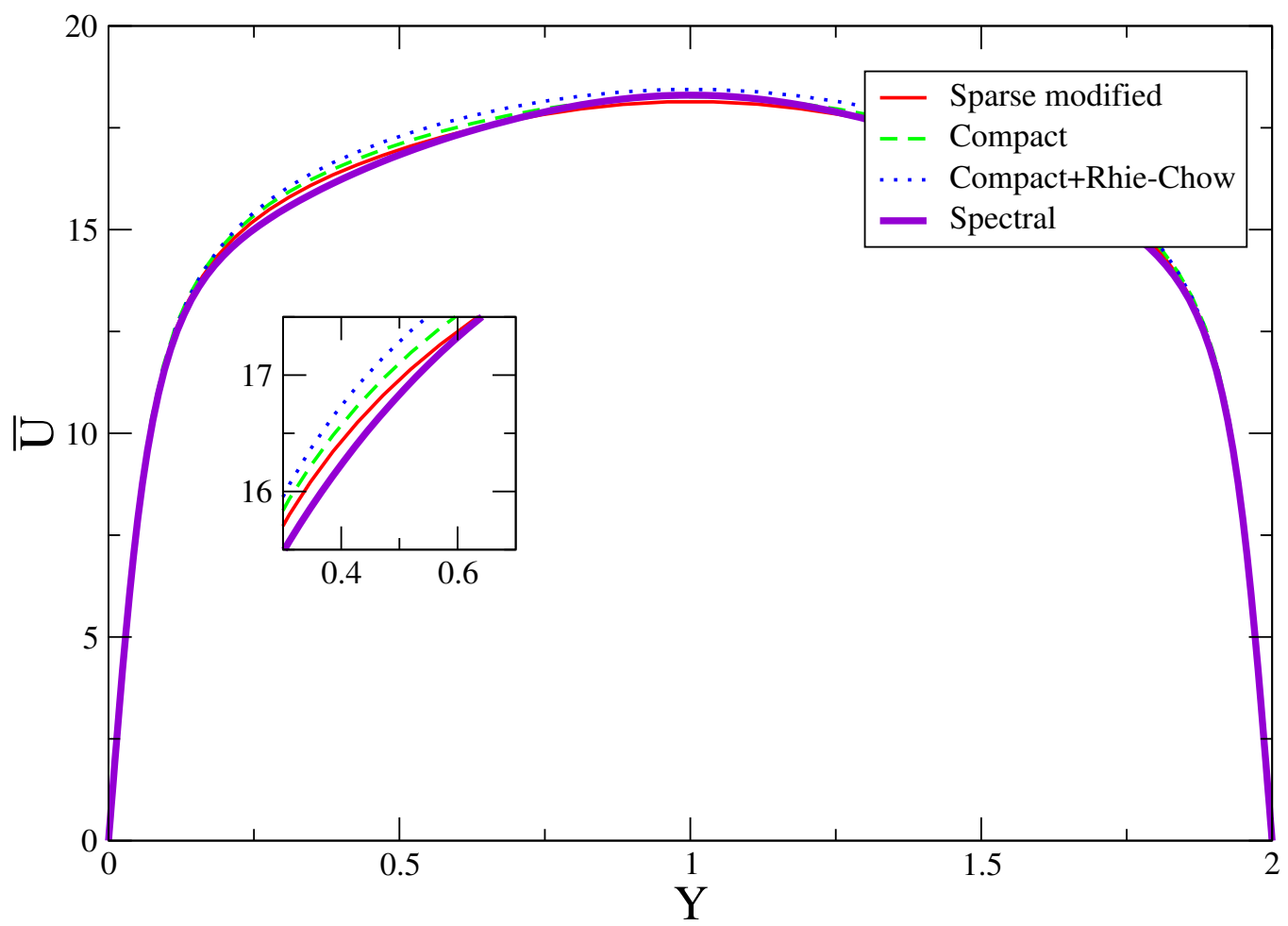

FiguRE 2: Staggered and non-staggered grid $\overline{\mathrm{U}}$ results compared to spectral benchmark, with magnified inset. 


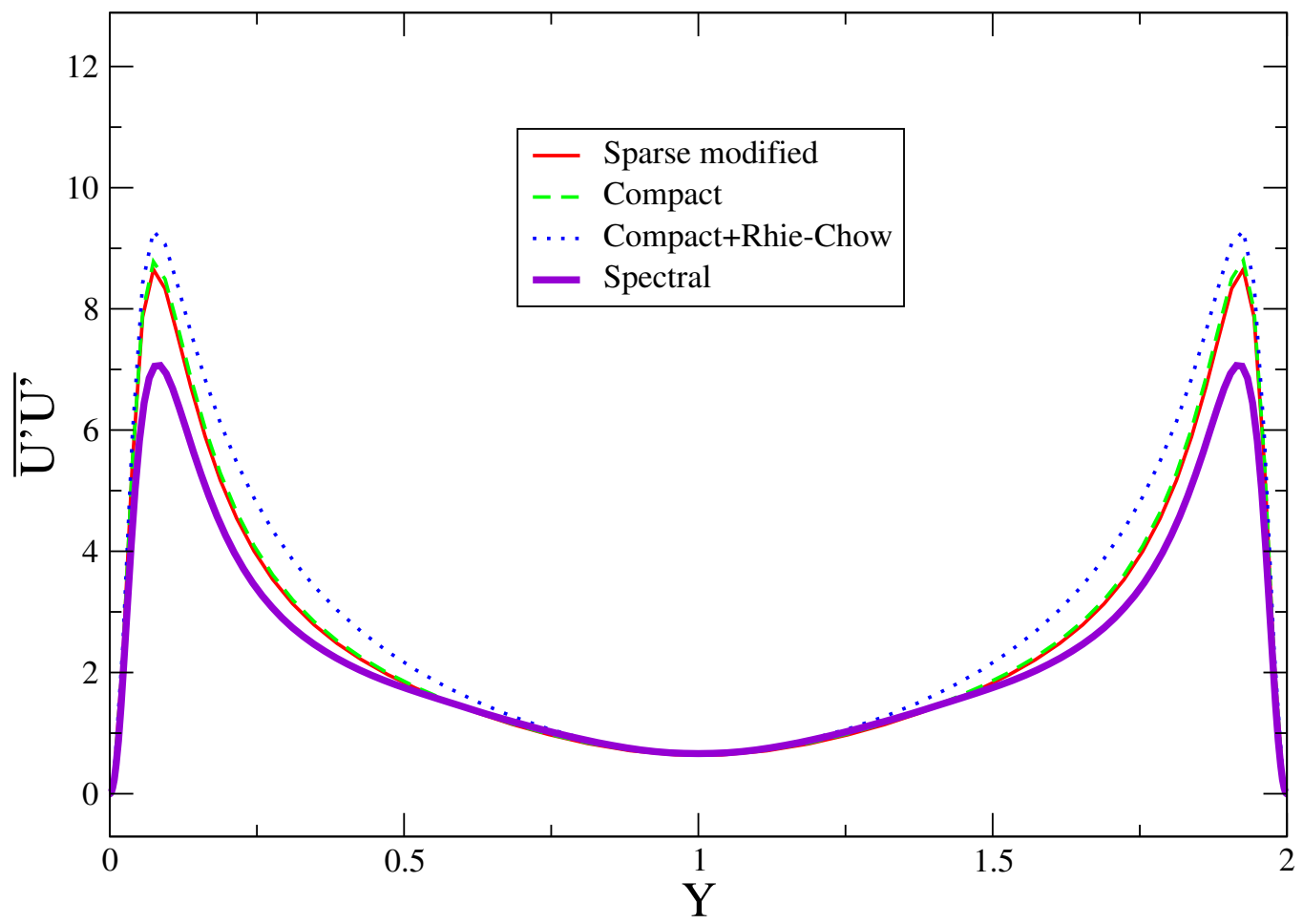

Figure 3: Staggered and non-staggered grid $\overline{\mathrm{U}^{\prime} \mathrm{U}^{\prime}}$ results compared to spectral benchmark. 
Acknowledgments The support of the Australian Research Council, and for R. Street the National Science Foundation (USA), are greatly appreciated.

\section{References}

[1] S. Armfield. Finite difference solutions of the Navier-Stokes equations on staggered and non-staggered grids. Computers and Fluids, 20:1-17, 1991. C655

[2] J. C. Strikewerda. Finite difference methods for the Stokes and Navier-Stokes equations. SIAM J. Sci. Stat. Comput., 5:56-68, 1984. $\mathrm{C} 655$

[3] S. Abdallah. Numerical solutions of the pressure Poisson equation with Neumann boundary conditions using a non-staggered grid. 1. J. Comp. Phys., 70:182-192, 1987. C655

[4] G. Schneider and M. Raw. Control volume finite-element method for heat transfer and fluid flow using collocated variables. 1.

Computational procedure. Numerical Heat Transfer, 11:363-390, 1987. C655

[5] C. M. Rhie and W. L. Chow. A numerical study of the turbulent flow past an airfoil with trailing edge separation. AIAA-82-0988, 1982. C655, C660

[6] S. W. Armfield and R. Street. Fractional step methods for the Navier-Stokes equations on non-staggered grids. ANZIAM $J$. 42(E):C134-C156, 2000. http://anziamj . austms .org.au/ojs/ index.php/ANZIAMJ/article/view/593. C660

[7] N. Williamson, S. W. Armfield and W. Lin. Transition behavior of weak turbulent fountains, J. Fluid Mech., 655:306-326, 2010. C660 
[8] R. D. Moser, J. Kim and N. N. Mansour. DNS of turbulent channel flow up to $\mathrm{Re}_{\tau}=590$. Phys. Fluids, 11:943-945, 1999. C662

\section{Author addresses}

1. S. W. Armfield, School of Aerospace, Mechanical \& Mechatronic Engineering, Sydney University, Sydney, Australia. mailto:armfield@aeromech.usyd.edu.au

2. N. Williamson, School of Aerospace, Mechanical \& Mechatronic Engineering, Sydney University, Sydney, Australia.

3. M. P. Kirkpatrick, School of Aerospace, Mechanical \& Mechatronic Engineering, Sydney University, Sydney, Australia.

4. R. Street, Environmental Fluid Mechanics Laboratory, Stanford University, Stanford, California 94305-4020, USA. 